\title{
Moderation role of attitude on the relationship between participation in competitive sports and academic performance of student-athletes in Saudi Arabia
}

\begin{abstract}
Background: This study aims to investigate how the attitude of undergraduate studentathletes moderates the relationship between competitive sports participation (CSP) and academic performance (AP). In this study, a type of attitudes was taken into consideration namely academic performance attitude (APT). Methods: A total of 102 undergraduate student athletes from 3 academic institutions in the Eastern Region of Kingdom of Saudi Arabia (KSA) were selected as subjects. Instruments used were Survey of Study Habits \& Attitudes (SSHA) by Brown and Holtzman, to obtain the data on APT. The instrument was backtranslated to the Arabic language in order to improve its comprehensiveness. Data on sports participation and academic performance were obtained by directly enquire the participants about their grade point average (GPA) and the frequency of their CSP in a self-developed questionnaire. Results: The results showed that APT significantly alters the effect of CSP on AP, because the 6 value of the effect of CSP on AP was greater than the 6 value of the effect of CSP and APT on AP. Because 6 value shows the strength of the influence, it can be concluded that the actual strength of the CSP is not as strong as when it is combined with APT. In other words, APT moderates the influence of CSP on AP. Discussion: The moderation effect occurs due to the regulation of Saudi Universities Sports Federation (SUSF) that only student-athletes with required academic scores can participate in competitive sports. Without such a regulation, APT might not be correlated to the AP because some students might pay more focus on CSP and less on AP. It can be concluded that the regulation helped student-athletes in KSA to improve their AP without sacrificing their CSP and vice versa.
\end{abstract}

Keyword: Sports participation; Academic performance; Competitive sports; Moderation; Attitude 